The Astrophysical Journal, 224:293-301, 1978 September 1

(C) 1978. The American Astronomical Society. All rights reserved. Printed in U.S.A.

\title{
ON THE FORMATION AND EVOLUTION OF CLUMPS OF GALAXIES IN AN EXPANDING UNIVERSE
}

\author{
C. A. NORMAN* AND JOSEPH SILK $\dagger$ \\ Received 1977 November 4; accepted 1978 March 7
}

\begin{abstract}
We derive results for the development of phase-space clumps of mass points in a background spectrum of gravitational potential fluctuations. The Vlasov equation and the pair correlation equation (in the weak coupling limit) are solved exactly in an Einstein-de Sitter cosmology, and the plasma clumping theory is used to identify terms that yield important collective effects. Various astrophysical implications are discussed, including the formation of large-scale inhomogeneity and the enhanced generation of correlations in the distribution of galaxies.
\end{abstract}

Subject headings: cosmology - galaxies: formation

\section{INTRODUCTION AND SUMMARY}

Clump formation has been observed in simulations of electrostatic plasmas. Test electrons with a neutralizing background are found to clump or group together in the presence of a background spectrum of electrostatic waves. These were not self-consistent simulations, in that Poisson's equation was not solved, but the background distribution of waves was specified.

We shall argue that similar results are valid for a gravitational problem where we consider test mass points moving in a background spectrum of gravitational potential fluctuations. The basis for the analogy is that, in both cases, identical equations of motions are obtained for the test particles. There is no a priori reason to suspect that a similar result is even qualitatively applicable to the case of a self-gravitating ensemble of particles, because, as is well known, the nonlinear self-consistent regime of the gravitational problem leads to secular growth. We are able to apply Dupree's (1972) theory of clump formation, which can be justified on the basis that it gives a reasonable description of the plasma simulation experiments, to the case of test particles (galaxies) moving in a spectrum of waves determined by other considerations (such as the propagation of potential fluctuations or sound waves in a hot background gas or matter distribution).

It is nontrivial to take these results over to the case of an expanding, self-gravitating system of point masses, as required for the self-consistent cosmological problem. The reason is that at least the linearized eigenfunctions of the system of point masses must be specified in order to evaluate the quasi-linear diffusion coefficient required by Dupree's theory. While in the nonexpanding case the eigenfunctions are simple waves, we find that, when expansion and self-gravity are introduced, secular modes are obtained for wavelengths large compared to the Jeans length.

To get around this difficulty, we have found an analytic solution of the linearized Vlasov equation in a cosmological background, and we are able to define a phase velocity for the resulting eigenfunctions. This enables us to understand how test particles can interact with these modes in a resonant fashion as required by the theory of clump formation. It should be noted that this resonance effect is an extension of standard quasi-linear theory to include phase-space granulation. One specific application of this result is to the formation of clumps of test particles in the presence of a hot background. Clumping occurs only for test particles with a narrow range of velocity dispersion, and the crossing time (over which the clumps will disperse) may therefore be relatively long. These cold clumps (which we may identify with systems spanning the range from groups to superclusters of galaxies) consist only of test masses: the question of their gravitational binding is dependent on an appropriate choice of mass. Some of these systems could therefore, at least in principle, be unbound.

We do not attempt here to solve the initial value problem for clump formation. Dupree, Wagner, and Mannheimer (1975) have some confidence in the rapid formation of clumps from $N$-body simulations. Similar calculations for a self-gravitating system of expanding mass points should ultimately be capable of resolving this issue for the present problem. On scales smaller than the Jeans length, expansion is unimportant, and the arguments for clumping of test particles in a background of potential fluctuations are valid.

The physical nature of the clumping process can be clarified by means of arguments similar to those of Dupree. Because we are dealing with a collisionless Vlasov equation, the density of individual fluid elements in phase space is conserved. Clump formation occurs because fluid elements with very different phase-space densities can be associated. Situations can arise where clumps form in phase space with sufficiently narrow widths that trapping

* Huygens Laboratory, Leiden.

$\uparrow$ Astronomy Department, University of California, Berkeley. 
in the resonant background spectrum of potential fluctuations will prolong the clump lifetimes. It may not be obvious that such resonant processes will occur in the case of an expanding distribution of test masses; however, our analysis of the linearized Vlasov equation and extension to clumping theory clearly demonstrates the existence of such effects. We are of course assuming the existence of a spectrum of waves with the required phase velocities that are indicated by our solutions of the linearized Vlasov equation. A possible astrophysical source of such waves would be sound waves generated in a hot gas by radio galaxy explosions; however, we know of no detailed calculations which allow us to estimate the spectrum and energy density of such waves. Another possibility would be gravitational potential fluctuations generated during the process of galaxy and cluster formation. The latter process is similar to that of violent relaxation, except that we have now included the possibility of clump formation. Such clumps, once formed, can drive the system to violent relaxation over a relatively rapid time scale.

Another application of our solution of the Vlasov equation is to the Balescu-Lenard equation for the two-point correlation function. We are able to solve this equation in the weak coupling limit, and obtain results consistent with those obtained previously by other authors.

Once clumps develop, the evolution of the two-point correlation can be enhanced at a rate considerably in excess of that expected from linear theory. The work of Kadomtsev and Pogutse (1970) suggests that the growth rate is proportional to the clump mass. A possible application is the recent work on $N$-body simulations of galaxy clustering.

We shall first describe the evolution of the distribution function of self-gravitating mass points in a given cosmological model using the linearized Vlasov equation. This problem has been previously discussed by Gilbert (1966) and Bisnovaty-Kogan and Zel'dovich (1971), who solved the linearized Vlasov equation and found evidence for Landau damping phenomena. We shall investigate the problem using suitable frequency and wavenumber variables ( $(\mathrm{II})$, and then give in detail the self-gravitating analogue of the quasi-linear work of Dupree (1972) $(\S$ III).

We shall extend these results to include the effects of a hot background intergalactic gas or other cosmological substratum which dominates the matter density of the universe ( $§$ IV). In this case we are looking at the motion of the galaxies, which can be visualized as test particles in a background system of waves. In contrast to the previous case, the characteristics of the waves are determined solely by the assumed background parameters rather than by the properties of the observed distribution of galaxies. We assume that the potential fluctuations due to the presence of the galaxies are small compared to the background waves; this is clearly possible when the mass density of the background dominates that due to the individual galaxies. We propose that a spectrum of sound waves is generated in the background fluid at the epoch of galaxy formation, and we shall estimate the effect of these waves on the clumping of galaxies.

The above ideas are also of interest in the study of the dynamics of groups, clusters, and superclusters of galaxies, and we consider a variety of astrophysical applications in $\S$ IV.

We shall attempt to relegate the more intricate mathematical details to the Appendices and only include them where we require to illustrate various physical arguments.

\section{THE LINEARIZED VLASOV EQUATION}

It is necessary to know the eigenmodes of our system if we are to investigate collective effects. A knowledge of these linear eigenmodes and their space-time Fourier transforms enables us to look for resonant effects in the transformed variables (which we define later). Specifically if one has an initially cold distribution of mass points, the unstable modes can still be randomly phased corresponding to their initial amplitudes and give resonance effects analogous to Landau damping. Following the work of Bisnovaty-Kogan and Zel'dovich (1971), we shall look for solutions of the linearized Vlasov equation in an Einstein-de Sitter universe with scale factor $a(t) \propto t^{2 / 3}$. The linearized Vlasov equation is

$$
\frac{\partial f^{(1)}}{\partial t}+v \cdot \frac{\partial f^{(1)}}{\partial x}+\frac{\partial \phi}{\partial x} \cdot \frac{\partial f_{0}}{\partial v}=0
$$

where $f^{(1)}$ is the perturbed portion of the distribution function, $f_{0}$ is the unperturbed background distribution function, $\phi$ is the perturbed gravitational potential fluctuation, and $(\boldsymbol{x}, \boldsymbol{v})$ are phase-space coordinates of a galaxy in position and velocity. The problem is made self-consistent by using Poisson's equation

$$
\nabla^{2} \phi=4 \pi G \rho^{(1)}
$$

where $\rho^{(1)}=\int f^{(1)} d^{3} v$ is the perturbed density fluctuation. We choose as initial conditions $f^{(1)}=0$ at $t=0$ and let the background distribution function have the Maxwellian form

$$
f_{0}=\frac{1}{6 \pi G m} \frac{1}{(\pi \theta)^{3 / 2}} \exp \left(-u^{2} / \theta^{2}\right)
$$


where $G$ is the Newtonian gravitational constant, $m$ is the mass of a galaxy, $\theta$ is the rms random velocity of a galaxy relative to the Hubble expansion, and

$$
\boldsymbol{u}=a(t)(\boldsymbol{v}-H \boldsymbol{x}),
$$

where $H$ is the Hubble constant. The solutions to (1) and (2) are given in Appendix A, where for simplicity the perturbation has been restricted to be along one of the coordinates $x_{i}$.

It is usual in plasma physics to find that the linearized Vlasov equation leads to a dispersion relation relating frequency and wavenumber. In the present case, because of the time dependence of the coefficients introduced by the cosmological model, we do not obtain a simple algebraic relation using frequency and wavenumber variables, but we instead have found the second order differential equation

$$
\frac{\partial^{2} S}{\partial y^{2}}+12[1+y Z(y)] S=0,
$$

where

$$
t^{4 / 3} \phi(x, t)=\frac{1}{(2 \pi)^{4}} \int d^{3} k \int d \omega \exp \left[i\left(\omega t^{-1 / 3}+k_{x} x t^{-2 / 3}\right)\right] S(\omega),
$$

$Z(y)$ is the well studied plasma dispersion function (Fried and Conte 1961), and $y=\omega / k_{x} \theta$. At large $y$, we have $S(y) \sim y^{3}, y^{-2}$; and for small $y$ we have $S(y) \sim \exp \left( \pm 12^{1 / 2} y\right)$. These solutions correspond to the well-known growth and decay modes at wavelengths large compared to the Jeans length, and to damping modes at wavelengths short compared to the Jeans length (Biznovaty-Kogan and Zel'dovich 1971).

\section{PAIR CORRELATIONS}

Using the Vlasov cumulant hierarchy (Davidson 1972), Inagaki (1976) derived the fluid limit of the BBGKY hierarchy in a cosmological model and examined collective effects in clustering of galaxies. In his analysis, Inagaki ignored both resonance effects and the effects of clumps on the evolution of the distribution function. As we mentioned in $\S \mathrm{I}$, resonance effects can lead to significant clumping of particles in phase space. We now further elucidate this point for the clustering of galaxies. Our analysis must be extended to the highly nonlinear regime by numerical simulations. One of our major objectives is to demonstrate that studies of $N$-body simulations of galaxy clustering should reveal the occurrence of collective effects in phase space.

We neglect the three-particle correlation function and concentrate on identifying the physical processes in formation of the pair correlation function. The formulation and solution of this problem is given in Appendix B, and the result can be shown as follows:

$$
\langle f(1) f(2)\rangle=P_{\text {discrete }}+P_{\text {collective }}+P_{\text {resonant }},
$$

where the first two terms have been identified by Inagaki, and the resonant term requires special treatment.

\section{a) Clumping due to Resonant Processes}

The mathematical details, where we use the formalism of Dupree, Wagner, and Mannheimer (1975) that has been developed for resonant clumping in an electrostatic plasma and apply it to the cosmological problem, are left to Appendix C. However, there are several points worth noting about the physical processes. Let us first describe the basic physics of the clumping process, ignoring the cosmological background. Consider a clump of galaxies that is present initially. The galaxies tend to diffuse away from one another by interacting with the background fluctuations. The lifetime of a clump is of the order of magnitude of this diffusion time, which can be long if the clumping is in small regions of phase space. This time scale $t_{D}$ is given by

$$
t_{D} \approx\left[\frac{1}{3} k_{0}^{2} D(v)\right]^{-1 / 3}
$$

where $k_{0}$ is a characteristic wavenumber for the turbulence and $D$ is the quasi-linear diffusion coefficient describing the action of the wave turbulence on the particle motion in velocity space, given by

$$
D(v)=\frac{1}{4 \pi} \int d k k^{2}\left\langle\phi^{2}(k, \omega)\right\rangle_{\omega=k v},
$$

where the angular brackets denote a random phase average, $\phi(k, \omega)$ is the Fourier transform of the potential $\phi(x, t)$, and we have explicitly included the condition $\omega=k \cdot v$ (from standard quasi-linear theory of the Vlasov equation).

Significantly long lifetimes are expected only when the spatial extent of a clump is significantly less than the Jeans length $\lambda_{J}$ and the velocity spread in a clump is significantly less than the thermal velocity. The Fourier transform of the one-dimensional two-point correlation function has been calculated by Dupree (1972) to be 
$\sim 2 \pi k^{-2}\left[1-J_{0}\left(6^{1 / 2} k / k_{0}\right)\right]$. However, we shall not place any emphasis on its form because the observed two-point correlation function of galaxies is in the highly nonlinear regime where our analysis is not applicable. We do wish to emphasize that, according to the results of Dupree, Wagner, and Mannheimer (1975), clumps can have a twopoint correlation function that is at least an order of magnitude greater than the one calculated ignoring resonances for small phase-space volumes. These clumps can themselves additionally enhance the fluctuation level. We suggest here that clump formation has a significant influence on clustering of galaxies.

\section{b) Clustering in a Cosmological Context}

The above discussion must be adapted to take account of cosmological expansion. The concept of ensemble average must be generalized to include an average taken over the set of all possible universes. This concept can be justified in the context of Newtonian cosmology (cf. Olson and Sachs 1973). As is shown in Appendix C, the entire theory can now be carried over into a cosmological situation by changing variables to $\tau=t^{-1 / 3}$ and $\xi=t^{2 / 3} x$. In terms of the new time coordinate $\tau$, the diffusion time is

where

$$
\tau_{D}=\left[\frac{1}{3} k_{0}^{2} D(u)\right]^{-1 / 3},
$$

$$
D(u)=\int d k k^{2}\left\langle\psi^{2}(k, \omega)\right\rangle_{\omega=k u}
$$

and

Now

$$
\psi(x, t)=t^{4 / 3} \phi(x, t)
$$

$$
\psi(k, \omega)_{\omega=k u}=\frac{\delta(k, \omega)_{\omega=k u}}{1+(u / \theta) Z(u / \theta)},
$$

where $\delta(k, \omega)$ denotes the Fourier transform of the density contrast, and so

$$
D(u)=\int d k k^{2} \frac{\{\delta(k, \omega)\}_{\omega=k u}^{2}}{1+(u / \theta) Z(u / \theta)} .
$$

Thus from equation (3), the diffusion time in the Einstein-de Sitter cosmology is proportional to $\frac{1}{3} k_{0}^{2} D(u)$, or from equation (5), to the level of turbulence $\delta(k, \omega)_{\omega=k v}$. This may be contrasted with the case where there is no cosmological evolution, where $\tau_{D}$ is inversely proportional to the one-third power of the level of turbulence.

\section{CLUMPING OF GALAXIES IN THE PRESENCE OF A BACKGROUND MEDIUM}

We now consider the physics of clumping of galaxies in the presence of a background spectrum of sound waves, whose dispersion relation is

$$
\omega^{2}=k^{2} v_{\mathrm{th}, B}^{2}-4 \pi G \rho_{B},
$$

where $v_{\mathrm{th}, B}$ is the rms velocity of a proton in the background medium and $\rho_{B}$ is the density of the background medium. The phase velocity of these waves $v_{\mathrm{ph}}$ is given by

$$
v_{\mathrm{ph}}^{2}=v_{\mathrm{th}, B}{ }^{2}\left(1-\frac{1}{k^{2} \lambda_{\mathrm{J}, B}{ }^{2}}\right),
$$

where $\lambda_{\mathrm{J}, B}$ is the Jeans length. The galaxies, which can be visualized as test particles moving in the background fluctuations, are assumed to have an initially Maxwellian distribution with rms velocity $v_{\text {th }, G}$. The quasi-linear diffusion coefficient can be written for test galaxies diffusing in these background waves as

$$
D(v) \approx \frac{\omega_{G, B}{ }^{3}}{k^{2}} \frac{1}{k \lambda_{\mathrm{J}}}\left\{\delta_{B(k, \omega)}\right\}^{2}{ }_{\omega=k v},
$$

where $\delta_{B}$ is the background density contrast and $\omega_{G, B}{ }^{2}=4 \pi G \rho_{B}$.

The characteristic diffusion time for a test galaxy in these waves is

$$
t_{D} \approx \frac{1}{3}\left[k^{2} D(v)\right]^{-1 / 3},
$$

where the quantity $\delta(k, \omega)_{\omega=k v}^{2}$ must be evaluated at $v_{\mathrm{ph}} \approx v_{\mathrm{th}, G}$. Thus

$$
t_{D} \approx \frac{1}{\omega_{G, B}}\left(\frac{\delta \rho_{B}}{\rho_{B}}\right)^{-2 / 3} .
$$


In Dupree's theory, the lifetime of a clump is

$$
t_{0} \approx \frac{1}{(12)^{1 / 3}} t_{D}
$$

where we consider only regions in phase space, with $k_{0} \Delta x<1, \Delta v<1 / k t_{0}$, and $k_{0}$ is a characteristic length scale in the turbulence.

The following crucial question remains: Can there exist a spectrum of oscillations in the hot background that will clump cold galaxies? This requires $v_{\mathrm{ph}}=v_{\mathrm{th}, G}$. If $v_{\mathrm{th}, G}{ }^{2}<v_{\mathrm{th}, B}{ }^{2}$, then $k^{2} \lambda_{\mathrm{J}}{ }^{2} \approx 1$, and thus we require a significant energy density in fluctuations close to the Jeans length. The width of clumping in velocity space is

$$
\frac{\Delta v}{v_{\mathrm{th}, G}} \lesssim \frac{\lambda_{\mathrm{J}}}{v_{\mathrm{th}, G} t_{0}} \approx\left[\delta_{B}\left(k \approx k_{\mathrm{J}}, \omega \approx \omega_{\mathrm{J}}\right)\right]^{2 / 3} .
$$

These estimates ignored the cosmological expansion. Using the results of $\S \mathrm{III}$, it is possible to show what effect this has, namely, that the diffusion time or clump lifetime will be proportional to $\left\{\delta_{B}(k, \omega)\right\}_{\omega=k u}$ rather than $\sim \delta(k, \omega)^{-2 / 3}{ }_{\omega=k u}$. The physics is the same as in $\S$ III, only one now requires significant fluctuations in the background near the Jeans length. Using the coordinates $\xi$ and $\tau$, we still expect to see significant phase-space clumping in $N$-body simulations in the presence of a dense, hot, turbulent background gas.

\section{ASTROPHYSICAL IMPLICATIONS}

We have argued that clumping of mass points in phase space can significantly affect the two-point correlation function. While we have not been able to consider the nonlinear regime (where the three-point function $\gg$ twopoint function $\gg$ one-point function), we have been able to isolate significant additional physical processes which indicate that collective effects are important. In particular, we urge those who are doing $N$-body simulations to look in phase space (coordinates $\xi$ and $\tau$ in this paper) for clumpiness. The time scale for relaxation is expected to be inversely proportional to the clump mass ${ }^{1}$ and should consequently depend on the initial conditions of such simulations. Such clumps may accelerate the early evolution of galaxy clusters, but tidal disruptions will tear them apart within a crossing time. Observations of long-lived clumps in phase space have already been observed in plasma simulations at levels well above the thermal, and we expect such effects to be present in the gravitational case.

It is currently an unresolved issue whether, in the case of the Local Supercluster, there are large deviations from the Hubble flow. If the Hubble flow is relatively uniform over scales of $\sim 100 \mathrm{Mpc}$, as argued by Sandage (1975), an explanation of the origin of large-scale inhomogeneities (Abell 1975; Shane 1975) becomes a pressing issue. This is particularly true if the universe is open, with the implication that gravitational instability and clustering is no longer operative at a redshift $z \lesssim 1 / \Omega$, where $\Omega$ is the ratio of the mean matter density to its closure value. If $\Omega \approx 1$, this difficulty is largely removed.

Clump formation may provide a natural means of obtaining large-scale structures in a low- $\Omega$ universe. For example, if $\Omega=0.1$, then gravitational instability allows at most an aggregation of $N \sim 10^{4}$ galaxies to develop from random statistical $\left(N^{1 / 2}\right)$ fluctuations at decoupling. Yet a typical large-scale inhomogeneity or supercluster extending over $\sim 100 \mathrm{Mpc}$ may contain $\gtrsim 10^{5}$ galaxies. Clump formation can boost the level of initial fluctuations by between one and two orders of magnitude (cf. III $a$ ), and play an important role in allowing large-scale fluctuations to develop (especially in models with $\Omega \lesssim 0.1$ ). One can also conceive of clump formation playing an important role in a closed universe. Provided that there is a dense, hot, and turbulent intergalactic medium, clumps will form in the galaxy distribution on scales less than the characteristic scale of turbulence. If we adopt characteristic parameters for such a plasma $\left(T_{8}=T / 10^{8} \mathrm{~K} ; n_{\mathrm{cr}}=3 \times 10^{-6} \Omega \mathrm{cm}^{-3}\right)$, the Jeans length amounts to $\sim 50 T_{8}{ }^{1 / 2} \Omega^{-1 / 2} \mathrm{Mpc}$ and provides an upper limit on the possible clump size. Large-scale turbulence may have been generated when the intergalactic gas was heated in the recent past by mechanisms such as radio galaxy explosions, whose energy input to a dense intergalactic gas $\left(\Omega=1\right.$ ) would suffice to maintain it at $\sim 10^{6} \mathrm{~K}$ (Ginzburg and Ozernoy 1966). It is of interest to note that the Jeans length may be of the order of the largest observed radio sources if $\Omega \approx 1$ and $T \approx 10^{6} \mathrm{~K}$; this would lead to the generation of large-amplitude sound waves capable of driving clumping of the galaxies. It seems possible that unbound clumps in the galaxy distribution may remain as the universe expands. The galaxies are trapped by diffusion in phase space in the background spectrum of waves, and the clump lifetimes can be relatively long. If the turbulence were to decay, the clumps would fly apart within a galaxy crossing time: this could be comparable to a Hubble time for a large-scale inhomogeneity.

We wish to acknowledge helpful comments on an early draft of this manuscript by G. Biznovaty-Kogan and S. M. Fall. One of us (C. A. N.) acknowledges the financial support of the Miller Institute, UC Berkeley, and the other (J. S.) acknowledges partial financial support from NSA grant NGR 05-003-578 and NSF grant AST 75-02181.

\footnotetext{
${ }^{1}$ This proportionality can be obtained from the kinetic equation for the evolution of the one-point distribution function given by Kadomtsev and Pogutse (1970), and can also be inferred from the study by Spitzer and Schwarzschild (1951) of stellar encounters with interstellar clouds.
} 


\section{APPENDIX A}

\section{SOLUTIONS OF THE LINEARIZED VLASOV EQUATION}

Following Bisnovaty-Kogan and Zel'dovich (1971), we assume eigenfunctions of the form $\phi \sim e^{i k \xi} \phi(t)$, $f \sim e^{i k \xi}$, and $\xi=x / t^{2 / 3}$ with a perturbation wavenumber $\boldsymbol{k}=(k, 0,0)$. The linearized Vlasov and Poisson equations give

$$
f=i k \frac{\partial f_{0}}{\partial u_{x}} \int_{0}^{t} \phi\left(t^{\prime}\right) \exp \left[3 i k u_{x}\left(t^{-1 / 3}-t^{\prime-1 / 3}\right)\right] d t^{\prime}
$$

and

$$
-k^{2} \phi(t)=\int f^{(1)} d^{3} v
$$

We then find an integral equation for the eigenfunction $\phi(t)$, namely,

$$
\phi(z)+6 z^{2} \int_{z}^{\infty} \frac{\phi\left(z^{\prime}\right)}{z^{\prime 4}}\left(z-z^{\prime}\right) \exp \left[-\alpha\left(z-z^{\prime}\right)^{2}\right] d z^{\prime}=0,
$$

where $\alpha=9 k^{2} \theta / 4$, and we have substituted $z=t^{-1 / 3}$. If we put $\psi=\phi(z) / z^{4}$, we obtain

$$
z^{2} \psi(z)+\int_{z}^{\infty} \psi\left(z^{\prime}\right) H\left(z-z^{\prime}\right) d z^{\prime}=0,
$$

where $H(z)=6 z \exp \left(-\alpha z^{2}\right)$. This is similar to a Volterra integral equation and can be solved by Laplace transform methods (Morse and Feshbach 1953). The integral equation can be transformed to a second order differential equation

$$
\frac{\partial^{2} \psi(p)}{\partial p^{2}}-G(p) \psi(p)=0
$$

where

$$
\psi(p)=\int_{0}^{\infty} e^{-p z} \psi(z) d z
$$

and

$$
G(p)=\frac{3}{\alpha}\left\{1+\pi^{1 / 2} \frac{p}{(4 \alpha)^{1 / 2}} \exp \left(p^{2} / 4 \alpha\right) \operatorname{Erfc}\left[-p(4 \alpha)^{-1 / 2}\right]\right\} .
$$

Let us consider the inversion of the Laplace transform

$$
\psi(z)=\frac{1}{2 \pi i} \int_{-i \infty+\delta}^{i \infty+\delta} e^{p z} \psi(p) d p
$$

Let $p=i y$ and $\psi(i p)=S(p)$; then

$$
\psi(z)=\frac{1}{2 \pi} \int_{-\infty}^{\infty} e^{i y z} S(y) d y .
$$

The differential equation for $S(y)$ becomes

$$
\frac{\partial^{2} S}{\partial y^{2}}+12[1+y Z(y)] S(y)=0,
$$

where $Z(y)$, the well-studied plasma dispersion function (Fried and Conte 1961), has the following properties at small and large $y$ :

$$
Z(y)=i \pi^{1 / 2} \exp \left(-y^{2}\right) \operatorname{erfc}(-i y),
$$

where, for small $y, Z(y)$ is equal to $i \pi^{1 / 2}-2+4 y^{2} / 3$, and for large $y, Z(y)=-1 / y-1 /\left(2 y^{3}\right)$. Hence for large $y$ we have

$$
\frac{\partial^{2} S}{\partial y^{2}}-\frac{6}{y^{2}} S(y)=0
$$


with solutions $S(y) \sim y^{3}, y^{-2}$, and for small $y$ we have

$$
\frac{\partial^{2} S}{\partial y^{2}}+12 S(y)=0,
$$

with solutions $S(y) \sim \exp \left( \pm i 12^{1 / 2} y\right)$. The beam plasma case can be treated in a similar manner.

It is also possible to formulate the problem in a different manner. We can transform the Vlasov equation as follows. The linearized Vlasov equation is

$$
\frac{\partial f^{(1)}}{\partial t}+\boldsymbol{v} \cdot \frac{\partial f^{(1)}}{\partial \boldsymbol{x}}+\frac{\partial \phi}{\partial \boldsymbol{x}} \cdot \frac{\partial f_{0}}{\partial \boldsymbol{v}}=0
$$

Here, let $f=f(\xi, v, \tau)$, where $\xi_{i}=x_{i} / t^{2 / 3}$ and $\tau=t^{-1 / 3}$; then

$$
\frac{\partial f}{\partial t}=\frac{\partial f}{\partial \xi_{i}} \frac{\partial \xi_{i}}{\partial t}+\frac{\partial f}{\partial \tau} \frac{\partial \tau}{\partial t}=\frac{\partial f}{\partial \xi_{i}}\left(-\frac{2}{3} x_{i} t^{-5 / 3}\right)+\frac{\partial f}{\partial \tau}\left(-\frac{1}{3} t^{-4 / 3}\right)
$$

and

$$
\frac{\partial f}{\partial x_{i}}=\frac{\partial f}{\partial \xi_{i}} t^{-2 / 3}
$$

Thus,

$$
\frac{\partial f}{\partial t}+v_{i} \frac{\partial f}{\partial x_{i}}=-\frac{1}{3} t^{-4 / 3}\left(\frac{\partial}{\partial \tau}-3 u \cdot \frac{\partial f}{\partial \xi}\right)
$$

where $u=a(t)(v-H x)$. Also

$$
\frac{\partial f_{0}}{\partial v}=\frac{\partial f_{0}}{\partial u_{i}} \frac{\partial u_{i}}{\partial v}=t^{2 / 3} \frac{\partial f_{0}}{\partial u}
$$

and

$$
\frac{\partial}{\partial x}\left(t^{4 / 3} \phi\right)=\frac{\partial}{\partial x} \psi(\xi, \tau)=\frac{\partial \psi}{\partial \xi_{i}} \frac{\partial \xi_{i}}{\partial x}=t^{-2 / 3} \frac{\partial \phi}{\partial \xi} .
$$

The transformed Vlasov equation becomes

$$
\frac{\partial f}{\partial \tau}-3 \boldsymbol{u} \cdot \frac{\partial f}{\partial \xi}-3 \frac{\partial \psi}{\partial \xi} \cdot \frac{\partial f_{0}}{\partial \boldsymbol{u}}=0
$$

Let us now introduce the Fourier transform

$$
f^{(1)}(\xi, \tau)=\frac{1}{(2 \pi)^{4}} \int d^{3} k \int d \omega e^{i(k \xi+\omega \tau)} f(k, \omega) .
$$

The transformed linearized Vlasov equation becomes

$$
f^{(1)}(k, \omega)=-\frac{3}{\omega-3 k u_{x}} k \frac{\partial f_{0}}{\partial u_{x}} .
$$

Poisson's equation reduces to

$$
k^{2} \frac{\partial^{2} \psi}{\partial \omega^{2}}=\left(12 \pi G \int d^{3} u \frac{1}{\omega-3 k u_{x}} k \frac{\partial f_{0}}{\partial u_{x}}\right) \psi=0,
$$

and thus we obtain the second order differential equation for $\psi$ :

$$
\frac{\partial^{2} \psi}{\partial \omega^{2}}+\left\{\frac{12 \pi G}{k^{2}} \int d^{3} u \frac{1}{\omega-3 k u_{x}} k \frac{\partial f_{0}}{\partial u_{x}}\right\} \psi=0 .
$$

This latter equation is equivalent to equation (A1), which we have already solved. 


\section{APPENDIX B}

\section{PAIR CORRELATION EQUATION}

The equation governing the behavior of the pair correlations $G\left(\boldsymbol{k}, u_{1}, u_{2}, \tau\right) \equiv\left\{f^{(1)}(1) f^{(1)}(2)\right\}$ in the weak coupling limit neglecting the three-point correlations is (Fall and Severne 1976; Inagaki 1976)

where

$$
\left[\frac{\partial}{\partial \tau}+L\left(\boldsymbol{k}, \boldsymbol{u}_{1}, \tau\right)+L\left(-\boldsymbol{k}, \boldsymbol{u}_{2}, \tau\right)\right] G=\frac{-i}{2 \pi^{2}} G m \frac{\boldsymbol{k}}{k^{2}}\left[F\left(\boldsymbol{u}_{2}, \tau\right) \frac{\partial F\left(\boldsymbol{u}_{1}, \tau\right)}{\partial \boldsymbol{u}_{1}}-F\left(\boldsymbol{u}_{1}, \tau\right) \frac{\partial F\left(\boldsymbol{u}_{2}, \tau\right)}{\partial u_{2}}\right],
$$

$$
L(k, u) G\left(k, u_{1}, u_{2}, \tau\right)=i k \cdot u_{1} G\left(k, u_{1}, u_{2}, \tau\right)+L \pi G \frac{i}{k^{2}} k \cdot \frac{\partial F}{\partial u_{1}}\left(u_{1}, \tau\right) \int d u_{3} G\left(k, u_{3}, u_{2}, \tau\right) a(\tau) .
$$

This equation is solved using a Green's function technique. If we introduce a Green's function (or propagator) $g\left(k, u_{1}, \tau ; u_{1}, \tau_{0}\right)$ by writing

$$
\begin{aligned}
G\left(k, u_{1}, u_{2}, \tau\right)= & \int d u_{1}{ }^{\prime} \int d u_{2}{ }^{\prime} g\left(k, u_{1}, \tau ; u_{1}{ }^{\prime}, \tau_{0}\right) g\left(-k, u_{2}, \tau ; u_{2}{ }^{\prime}, \tau_{0}\right) G\left(k, u_{1}{ }^{\prime}, u_{2}{ }^{\prime}, \tau_{0}\right) \\
& -\frac{i}{2 \pi^{2}} G m \int_{\tau_{0}}^{\tau} d \tau^{\prime} \int d u_{1}{ }^{\prime} g\left(k, u_{1}, \tau ; u_{1}{ }^{\prime}, \tau^{\prime}\right) g\left(-k, u_{2}, \tau ; u_{2}{ }^{\prime}, \tau^{\prime}\right),
\end{aligned}
$$

we can obtain the pair correlations in the usual manner by first solving for the propagator (Ichimaru 1975). The equation for the propagator is found to be (suppressing $\tau_{0}$, which we set equal to $-\infty$ )

$$
\left(\frac{\partial}{\partial \tau}-i k \cdot u_{1}{ }^{\prime}\right) g\left(k, u_{1}{ }^{\prime}, u_{2}, \tau\right)=\frac{4 \pi i}{k^{2}} k \cdot \frac{\partial f_{0}}{\partial u_{1}} \int d^{3} u_{3} a(\tau) g\left(k, u_{3}, u_{2}, \tau\right),
$$

where $a(\tau)=1 / \tau^{2}$. Changing variables to

$$
\Gamma\left(k, u_{1}, u_{2}, \tau\right)=\frac{g\left(k, u_{1}, u_{2}, \tau\right)}{\tau^{2}},
$$

we obtain

$$
\left(\frac{\partial}{\partial \tau}-i k \cdot u_{1}\right) \tau^{2} \Gamma\left(k, u_{1}, u_{2}, \tau\right)=\frac{4 \pi i}{k^{2}} k \cdot \frac{\partial f_{0}}{\partial u_{1}} \int d^{3} u_{3} \Gamma\left(k, u_{3}, u_{2}, \tau\right) ;
$$

and after Fourier transforming,

$$
\frac{d^{2}}{d \omega^{2}} \Gamma\left(k, u_{1}, u_{2}, \omega\right)=\frac{1}{\omega-k \cdot u_{1}} \delta\left(u_{1}-u_{2}\right)+\frac{4 \pi i}{k^{2}} \frac{1}{\left(\omega-k \cdot u_{1}\right)} k \cdot \frac{\partial f_{0}}{\partial u_{1}} \int d^{3} u_{3} \Gamma\left(k, u_{3}, u_{2}, \tau\right) .
$$

Define

and then after integrating we find that

$$
W\left(k, u_{2}, \omega\right)=\int d^{3} u_{1} \Gamma\left(k, u_{1}, u_{2}, \omega\right)
$$

$$
\frac{d^{2}}{d \omega^{2}} W\left(\boldsymbol{k}, \boldsymbol{u}_{2}, \omega\right)-\left(\frac{4 \pi i}{k^{2}} \int d^{3} \boldsymbol{u}_{1} \frac{1}{\omega-\boldsymbol{k} \cdot \boldsymbol{u}_{1}} \boldsymbol{k} \cdot \frac{\partial f_{0}}{d u_{1}}\right) W\left(\boldsymbol{k}, \boldsymbol{u}_{2}, \omega\right)=\frac{1}{\omega+\boldsymbol{k} \cdot \boldsymbol{u}_{2}} .
$$

This is, as expected, the Vlasov equation solved in Appendix A, with an additional source term. This is a modified Volterra equation and can be solved by standard methods (Morse and Feshbach 1953). Although, as mentioned before, the dispersion relation is no longer algebraic, a second order differential equation for the quantity $W$ must be solved because of the influence of the cosmological evolution. Let us assume we have solved for $W\left(k, \omega, u_{2}\right)$; then

$$
\Gamma\left(\boldsymbol{k}, \boldsymbol{u}_{1}, \boldsymbol{u}_{2}, \omega\right)=\frac{1}{\omega-\boldsymbol{k} \cdot \boldsymbol{u}_{1}} \delta\left(\boldsymbol{u}_{1}-\boldsymbol{u}_{2}\right)+\frac{4 \pi G}{k^{2}} \frac{1}{\omega-\boldsymbol{k} \cdot \boldsymbol{u}_{1}} \boldsymbol{k} \cdot \frac{\partial f_{0}}{\partial u_{1}} W\left(\boldsymbol{k}, \boldsymbol{u}_{2}, \omega\right) .
$$

The propagator $g$ is given by $g=\partial^{2} \Gamma / \partial \omega^{2}$, and the resulting pair correlation function is given by Ichimaru (1975):

$$
G\left(k, u_{1}, \boldsymbol{u}_{2}\right)=-\frac{4 \pi i G}{k^{2}} \int d^{3} \boldsymbol{u}_{1}{ }^{\prime} \int d^{3} \boldsymbol{u}_{2}^{\prime} \int d \omega\left[\boldsymbol{k} \cdot\left(\frac{\partial}{\partial \boldsymbol{u}_{1}^{\prime}}-\frac{\partial}{\partial \boldsymbol{u}_{2}^{\prime}}\right) f\left(\boldsymbol{u}_{1}{ }^{\prime}\right) f\left(\boldsymbol{u}_{2}{ }^{\prime}\right) g\left(k, \boldsymbol{u}_{1}, \boldsymbol{u}_{1}^{\prime}, \omega\right) g\left(-\boldsymbol{k}, \boldsymbol{u}_{2}, \boldsymbol{u}_{2}^{\prime}, \omega\right)\right] .
$$


Using a different technique, Inagaki (1976) has shown that the velocity averaged density contrast $\bar{\delta}(\boldsymbol{k}, t)=$ $\int d u_{1} \int d u_{2} G\left(k, u_{1}, u_{2}, t\right)$ can be written for long wavelengths and long times as

$$
\bar{\delta}(k, t)=\frac{3 G m}{100 \pi^{2}}\left(\frac{t}{t_{0}}\right)^{4}+\frac{9}{25} \Gamma(k)\left(\frac{t}{t_{0}}\right)^{4},
$$

where $\Gamma(k)$ is proportional to the spatial Fourier transform of the initial two-point correlation function. We write this as

$$
\bar{\delta}(k, t)=P_{\text {discrete }}+P_{\text {collective }} .
$$

However, there are also significant collective terms that do not appear when one evaluates only the principal parts of all integrals. The extra terms we shall call $P_{\text {resonant }}$ and consider in detail in the following Appendix and in the text.

\section{APPENDIX C}

\section{CLUMPING DUE TO RESONANT PROCESSES}

We shall now derive characteristic clump parameters for gravitational systems using Dupree's theory of clumping (Dupree 1972; Hui and Dupree 1975; Dupree, Wagner, and Mannheimer 1975). We shall restrict ourselves to the one-dimensional case. Using the results of Appendix A, we have

$$
f^{(1)}(k, \omega)=-\frac{3 \psi(k, \omega)}{\omega-3 k u_{1}} k \frac{\partial f_{0}}{\partial u_{1}}
$$

Using the ensemble-averaging procedure discussed in $\S$ III, we find the two-point correlation function to be (Dupree, Wagner, and Mannheimer 1975)

$$
\begin{aligned}
\left\langle f^{(1)}\left(\xi^{(1)}, u^{(1)}, \tau\right) f^{(1)}\left(\xi^{(2)}, u^{(2)}, \tau\right)\right\rangle & \equiv\left\langle f^{(1)}(1) f^{(1)}(2)\right\rangle \\
& =9 \frac{\partial f_{0}^{(1)}}{\partial u_{1}^{(1)}} \frac{\partial f_{0}^{(2)}}{\partial u_{1}^{(2)}} \frac{1}{(2 \pi)^{4}} \int d^{3} k e^{-i k \Delta \xi} \int \frac{\left\langle\psi^{2}(k, \omega)\right\rangle k^{2} d \omega}{\left(\omega-3 k u_{1}^{(1)}\right)\left(\omega-3 k u_{1}^{(2)}\right)},
\end{aligned}
$$

where $\Delta \xi_{1}=\xi_{1}{ }^{(1)}-\xi_{1}{ }^{(2)}$. The resonant part of this can be written

$$
\left\langle f^{(1)}(1) f^{(1)}(2)\right\rangle \approx\left[\frac{\partial f_{0}^{(1)}}{\partial u_{1}^{(1)}}\right]^{2} \delta\left(u_{1}^{(1)}-u_{1}^{(2)}\right) \frac{2 \pi}{k_{0}} D\left(\Delta \xi, u_{1}^{(1)}\right),
$$

where

and

$$
D\left(\Delta \xi, u_{1}^{(1)}\right) \approx D\left(0, u_{1}^{(1)}\right)\left[1-k_{0}{ }^{2}\left(\Delta \xi_{1}\right)^{2}\right]
$$

$$
D(u) \equiv D(0, u)=\int \frac{d k}{4 \pi} k^{2}\left[\psi(k, \omega)^{2}\right]_{\omega=k u} \exp \left(i k \Delta \xi_{1}\right)
$$

is the usual quasi-linear diffusion coefficient. The most important parameter is the diffusion time $\tau_{D}$, which is proportional to the clump lifetime. It can be shown that $\tau_{D} \sim\left[\frac{1}{3} k_{0}^{2} D(u)\right]^{-1 / 3}$. Note that if we transform back to real time variables, $t \sim \tau^{-3}$, we have the diffusion time in real time $t_{D} \sim \frac{1}{3} k_{0}^{2} D(v)$.

\section{REFERENCES}

Abell, G. O. 1975, in Galaxies and The Universe, ed. A. Sandage, M. Sandage, and J. Kristian (Chicago: University of Chicago Press), p. 601.

Bisnovaty-Kogan, G. S., and Zel'dovich, Ya. B. 1971, Soviet Astr.-AJ, 14, 758 .

Davidson, R. C. 1972, Methods in Nonlinear Plasma Theory (New York: Academic Press).

Davis, M., and Peebles, P. J. E. 1977, Ap. J. Suppl., 34, 425.

Dupree, T. H. 1972, Phys. Fluids, 15, 334.

Dupree, T. H., Wagner, C. E., and Mannheimer, W. M. 1975, Phys. Fluids, 18, 1167.

Fall, S. M., and Severne, G. 1976, M.N.R.A.S., 174, 241.

Fried, B. D., and Conte, S. D. 1961, The Plasma Dispersion Function (New York: Academic Press).

Gilbert, I. H. 1966, Ap. J., 144, 233.

Ginzburg, V. L., and Ozernoy, L. M. 1966, Soviet Astr.-AJ, 9, 726 .

C. A. Norman: Huygens Laboratory, University of Leiden, Wassenaarseweg 78, Leiden 2405, Netherlands JOSEPH SILK: Department of Astronomy, University of California, Berkeley, CA 94720
Hui, B. H., and Dupree, T. H. 1975, Phys. Fluids, 18, 235.

Ichimaru, S. 1975, Basic Principles of Plasma Physics (Reading, MA: Benjamin).

Inagaki, S. 1976, Pub. Astr. Soc. Japan, 28, 463.

Kadomtsev, B. B., and Pogutse, O. P. 1970, Phys. Rev.

Morse, P. M., and Feshbach, H. 1953, Methods of Theoretical Physics (New York: McGraw-Hill).

Olson, D. W., and Sachs, R. K. 1973, Ap. J., 185, 91.

Sandage, A. R. 1975, Ap. J., 202, 563.

Shane, C. D. 1975, in Galaxies and The Universe, ed. A. Sandage, M. Sandage, and J. Kristian (Chicago: University of Chicago Press), p. 647.

Spitzer, L., and Schwarzschild, M. 1951, Ap. J., 114, 385. Letters, 25, 1155. 